\title{
REFERENCES
}

1. Yu. K. Belayev, Continuity and Hölder's conditions for sample functions of stationary Gaussian processes, Proc. Fourth Berkeley Symp., Vol. 2, pp. 23-33, 1961.

2. S. M. Berman, A law of large numbers for the maximum in a stationary Gaussian sequence, Ann. Math. Statist. 33 (1962), 93-97.

3. E. V. Bulinskaya, On the mean number of crossings of a level by a stationary Gaussian process, Teor. Verojatnost. i Primenen. 6 (1961), 474-477.

4. H. Cramér, Random variables and probability distributions, Cambridge Tracts in Mathematics, Vol. 36, Cambridge University Press, Cambridge, 1937. 2nd ed. to appear in 1962.

5. G. A. Hunt, Random Fourier transforms, Trans. Amer. Math. Soc. 71 (1951), 38-69.

6. M. Loève, Probability theory, 2nd ed., Van Nostrand, Princeton, N. J., 1960.

Research Triangle InStitute

\section{ON THE MAXIMUM TRANSFORM AND SEMIGROUPS OF TRANSFORMATIONS}

\author{
BY RICHARD BELLMAN AND WILLIAM KARUSH
}

Communicated by Peter D. Lax, April 27, 1962

1. Introduction. The problem of determining the maximum of the function

$$
F\left(x_{1}, x_{2}, \cdots, x_{N}\right)=\sum_{i=1}^{N} g_{i}\left(x_{i}\right)
$$

over the domain $D_{N}$ defined by $\sum_{i=1}^{N} x_{i}=x, x_{i} \geqq 0$, is one with various ramifications and applications. Analytic solutions and computational algorithms have been obtained in a number of ways; see Karush [7], Bellman [2], Bellman and Karush [3]. Let us now discuss a new way of generating solutions of (1.1). Let $g(x, a)$ be a scalar function of the scalar variable $x$ and the $M$-dimensional vector $a$ with the group property that

$$
\max _{x_{1}+x_{2}=x}\left[g\left(x_{1}, a\right)+g\left(x_{2}, b\right)\right]=g(x, h(a, b)) \quad\left(x_{1}, x_{2} \geqq 0\right),
$$

where $h(a, b)$ is a known function of $a$ and $b$. It follows inductively that

$$
\max _{D_{N}}\left[\sum_{k=1}^{N} g\left(x_{k}, a^{(k)}\right)\right]=g\left(x, h\left(a^{(1)}, a^{(2)}, \cdots, a^{(N)}\right)\right),
$$

where $D_{N}$ is as above, and $h\left(a^{(1)}, a^{(2)}, \cdots, a^{(N)}\right)$ is obtained from 
$h(a, b)$ in a recurrent fashion. The function $g(x, a)=a x^{p}, 0<p \leqq 1$, with $a \geqq 0$ is a function of the desired type. How can we generate classes of functions with this property, and can we determine all of them?

2. The maximum transform. In previous papers $[3 ; 4 ; 5]$, we discussed the transform

$$
F(y)=M(f)=\max _{x \geqq 0}[f(x)-x y],
$$

a transform which plays a basic role in the study of convexity; see Fenchel [6], Beckenbach and Bellman [1]. This transform possesses the important dissolving property

$$
M\left[\max _{x_{1}+x_{2}=x}\left[g\left(x_{1}, a\right)+g\left(x_{2}, b\right)\right]\right]=M(g(x, a))+M(g(x, b)) .
$$

Taking advantage of this relation, we can obtain functions satisfying (1.2) by starting with functions $G(x, a)$ satisfying the simpler relation

$$
G(x, a)+G(x, b)=G(x, h(a, b)),
$$

and inverting

$$
g(x, a)=M^{-1}(G(x, a))=\min _{y \geqq 0}[G(y, a)+x y] .
$$

3. Solutions of the functional equation. If $a$ is an $M$-dimensional vector with components $a_{1}, a_{2}, \cdots, a_{M}$, a very simple class of solutions of (2.3) is given by

$$
\begin{aligned}
G(x, a) & =(a, G(x))=\sum_{i=1}^{M} a_{i} G_{i}(x), \\
h(a, b) & =a+b .
\end{aligned}
$$

Under various assumptions of analyticity, it may be shown that aside from inessential changes of variable, $a \rightarrow \phi(a)$, these are the only solutions. Rigorous proofs will be given subsequently.

4. Parametric representation. If it is possible to obtain the minimum value in (2.4) by means of differentiation, we obtain the parametric representation

$$
\begin{aligned}
g(x, a) & =G(y, a)+x y, \\
x & =-G_{y}(y, a) .
\end{aligned}
$$

Assuming that $G(x, a)$ is given by (3.1), we face the interesting prob- 
lem of determining the $a_{i}$ and $G_{i}(x)$ so as to fit a given function $g(x)$ in some optimal fashion. Having done this, we can find quick and useful solutions to the original variational problem, (1.1) et seq. The point is that in this way we find exact solutions to approximate problems as opposed to the usual approximate solution to an exact problem.

In subsequent papers, we shall discuss the multidimensional and continuous versions of these problems and techniques.

\section{REFERENCES}

1. E. F. Beckenbach and R. Bellman, Inequalities, Ergebnisse der Math., Springer, Berlin, 1961.

2. R. Bellman, Dynamic programming, Princeton Univ. Press, Princeton, N. J., 1957.

3. R. Bellman and W. Karush, On a new functional transform in analysis: the maximum transform, Bull. Amer. Math. Soc. 67 (1961), 501-503.

4. R. Bellman, The maximum transform. I, Technical Memorandum TM-665, System Development Corp., Santa Monica, November, 1961.

5. - The maximum transform. II, Technical Memorandum TM-689, System Development Corp., Santa Monica, February, 1962.

6. W. Fenchel, On conjugate convex functions, Canad. J. Math. 1 (1949), 73-77.

7. W. Karush, $A$ general algorithm for the optimal distribution of effort, Management Sci., to appear; also available as Technical Memorandum TM-616, System Development Corp.

The RAND Corporation and

System Development Corporation 